Reprod. Nutr. Dévelop., 1983, 23 (3), 587-597.

\title{
Influence du remplacement d'une partie du suif d'un aliment d'allaitement par de la tricaproïne ou de l'huile de coprah, sur l'utilisation de l'énergie et de l'azote par le veau préruminant : influence du niveau d'alimentation antérieur
}

\author{
B. AUROUSSEAU, M. VERMOREL, J. C. BOUVIER
}

Avec la collaboration technique de F. DUBOISSET, Marinett MEYER, S. GASNET, G. MOINS et R. SOUCHET

Laboratoire d'Etude du Métabolisme énergétique, I.N.R.A., Theix, 63110 Beaumont, France.

Summary. Effect on energy and nitrogen balances of replacing part of the tallow in a milk replacer for preruminant calves by tricaproin or coconut oil : effect of previous feeding level.

Using two open-circuit respiratory chambers, the effects of medium chain fatty acid intake on digestibility, energy and nitrogen balance were investigated in 4 groups of preruminant Friesian male calves. One group of 7 animals was fed a control diet with a skim-milk powder and tallow base (diet 1). The other three groups were fed diets in which one-third of the tallow was replaced by either $1 / 3$ coconut oil (6 animals, diet 2 ) or $1 / 3$ tricaproin $(6$ animals, diet 3$)$. In diet 4 , two-thirds of the tallow was replaced by $1 / 3$ coconut oil $+1 / 3$ tricaproin (5 animals).

Apparent digestibility and energy metabolizability (q) were similar for diets 1, 2, 3 and significantly higher $(P<0.05)$ for diet $4: 97.5$ vs $95.1 \%$ for dry matter digestibility, 97.3 vs $94.5 \%$ for energy digestibility, 95.6 vs $93.7 \%$ for crude protein digestibility and 94.6 vs $92.8 \%$ for $\mathrm{q}$.

Whatever the diet, metabolizable energy (ME) efficiency for tissue deposition was $70 \pm 9 \%$ and maintenance requirements amounted to an average of $90 \mathrm{kcal}$ $\mathrm{ME} / \mathrm{d} / \mathrm{kgW} \mathrm{W}^{0.75}$. However, the latter were related to previous feeding level and increased by $0.13 \pm 0.06 \mathrm{kcal} / \mathrm{d} / \mathrm{kgW}^{0.75}$ for an increase of $1 \mathrm{kcal} / \mathrm{d} / \mathrm{kgW}^{0.75}$ of previous ME intake.

The lipids in each of the 4 diets were also deposited at a rate of $0.40 \pm 0.09 \mathrm{kcal}$. Lipid gain was decreased by $0.12 \pm 0.08 \mathrm{kcal}$ for an increase of $1 \mathrm{kcal} / \mathrm{d} / \mathrm{kg} W^{0.75}$ of previous Me intake.

The regression coefficients of protein energy gain on Me intake were $0.25 \pm 0.01$; $0.25 \pm 0.06 ; 0.30 \pm 0.04$ and $0.29 \pm 0.06 \mathrm{kcal} \mathrm{PF} / \mathrm{kcal}$ of $\mathrm{ME}$, respectively, for " tallow ", " tallow-coconut oil ", " tallow-TC6 " and " tallow-coconut oil-TC6 " diets ; protein gain did not depend on the previous level of ME intake.

Finally, for each of the four diets, respective adjusted energy gains were $107.5^{\mathrm{a}}$; $122.6^{\mathrm{b}} ; 105.6^{\mathrm{a}}$ and $99.1^{\mathrm{a}} \mathrm{kcal} / \mathrm{d} / \mathrm{kgW}$.75, adjusted lipid gains $60.4^{\mathrm{a}} ; 69.0^{\mathrm{a}} ; 50.9^{\mathrm{b}}$ and $42.6^{\mathrm{c}} \mathrm{kcal} / \mathrm{d} / \mathrm{kgW}^{0.75}$ and adjusted protein gains $47.6^{\mathrm{a}} ; 53.6^{\mathrm{b}} ; 53.8^{\mathrm{b}}$ and $57.5^{\mathrm{c}}$ $\mathrm{kcal} / \mathrm{d} / \mathrm{kgW}$. .75 (data with a different superscript are significantly different ; $\mathrm{P}<0.05$ ).

In the present study, the energy cost of protein deposition amounted to $2.29 \pm 0.21$ $\mathrm{kcal} \mathrm{ME} / \mathrm{kcal}$ of protein and that of lipid deposition to $0.99 \pm 0.20 \mathrm{kcal} \mathrm{ME} / \mathrm{kcal}$ of lipid. 


\section{Introduction.}

Les capacités de digestion des matières grasses conventionnelles (essentiellement le suif) sont limitées à la naissance du Veau (Stobo et Roy, 1977). Par ailleurs, cet animal ne tolère pas des apports trop importants de glucides solubles (Mathieu et De Tugny, 1965 ; Lodge et Lister, 1973) et ne peut utiliser efficacement l'amidon avant l'âge de 4 à 9 semaines (Mathieu et Thivend, 1968). En revanche, des suppléments d'énergie semblent pouvoir lui être apportés dès la naissance par les acides gras à chaîne moyenne (AGCM) qu'il digère avec une grande efficacité (Raven et Robinson, 1958 ; Niesar, 1964 ; Toullec et Mathieu, 1969 ; Veen, 1970b). De plus, l'ingestion d'acide caproïque permet une augmentation de la fixation de protèines chez le Rat prépubère (Chenat et al., 1976) tandis que l'ingestion d'huile de coprah peut améliorer la vitesse de croissance du Veau préruminant (Raven et Robinson, 1964 ; Veen, 1970a ; Roy et al., 1973). II était donc intéressant de préciser les effets de l'ingestion d'huile de coprah ou de tricaproïne (TC6) et l'influence du niveau d'alimentation sur l'utilisation digestive et métabolique de l'énergie et de l'azote de l'aliment d'allaitement par le très jeune Veau préruminant.

\section{Matériel et méthodes.}

\section{Dispositif expérimental.}

Quatre laits iso-énergétiques et iso-azotés comportant $27 \%$ de protéines et $22.6 \%$ de matières grasses (dont 2,5\% d'émulsifiant) ont été préparés (tabl. 1) :

TABLEAU 1

Composition et caractéristiques des aliments d'allaitement

\begin{tabular}{|c|c|c|c|c|}
\hline & «Suif » & « Suif-coprah » & "Suif-TC6 » & $\begin{array}{l}\text { "Suif-coprah- } \\
\text { TC6» }\end{array}$ \\
\hline \multicolumn{5}{|l|}{ Composition (g/kg MS) } \\
\hline Poudre de lait écrémé & 753,2 & 752,2 & 750,5 & 747,9 \\
\hline Suif $\ldots \ldots \ldots \ldots \ldots$. & 201,2 & 113,3 & 86,3 & - \\
\hline Huile de coprah $\ldots \ldots \ldots \ldots \ldots \ldots$. & - & 88,9 & - & 88,9 \\
\hline Suif-TC6 (intérestérifiés) . . . . . . . & - & - & 117,6 & 117,6 \\
\hline Emulsifiant $\ldots \ldots \ldots \ldots \ldots$ & 24,9 & 24,9 & 24,9 & 24,9 \\
\hline Composé minéral et vitaminique & 20,7 & 20,7 & 20,7 & 20,7 \\
\hline \multicolumn{5}{|l|}{ Caractéristiques } \\
\hline $\begin{array}{l}\text { Energie (kcal/g MS) } \\
\text { Azote }(\mathrm{g} \mathrm{N} / \mathrm{g} \mathrm{MS})\end{array}$ & $\begin{aligned} 5480 \\
\quad 44,9\end{aligned}$ & $\begin{array}{l}5486 \\
45,2\end{array}$ & $\begin{array}{r}5335 \\
\quad 45,4\end{array}$ & $\begin{array}{l}5245 \\
\quad 44,2\end{array}$ \\
\hline Lipides (g / kg MS). & 224 & 226 & 218 & 214 \\
\hline \multicolumn{5}{|l|}{$\begin{array}{r}\text { Nature des acides gras alimentaires } \\
(\% \text { AG totaux })\end{array}$} \\
\hline Acide caproïque $\ldots \ldots \ldots \ldots \ldots$ & - & - & 34,0 & 34,0 \\
\hline Acides laurique et myristique ...... & 4,2 & 12,7 & 2,7 & 22,8 \\
\hline Acides palmitique et stéarique ..... & 49,6 & 43,2 & 32,3 & 22,8 \\
\hline Acide olé̈que...$\ldots \ldots \ldots \ldots$ & 36,8 & 35,6 & 23,6 & 13,9 \\
\hline Acide linoléique $\ldots \ldots \ldots \ldots \ldots$ & 3,5 & 3,3 & 3,2 & 3,2 \\
\hline
\end{tabular}


a) un lait témoin "suif » à base de suif et de poudre de lait écrémé ;

b) un lait " suif-coprah " obtenu par remplacement de $1 / 3$ du suif par de I'huile de coprah ;

c) un lait « suif-TC6 » obtenu par remplacement de $1 / 3$ du suif par de la tricaproïne ;

d) un lait "suif-coprah-TC6 " obtenu par remplacement de $2 / 3$ du suif, pour moitié par de I'huile de coprah et pour moitié par de la tricaproïne.

La tricaproïne était introduite dans les deux derniers aliments sous forme intérestérifiée à du suif.

L'étude a porté sur 24 veaux mâles de race Frisonne, achetés à un âge de 4 à 8 jours. Les animaux ont été placés en cases individuelles et affectés dès leur arrivée à l'un des traitements décrits ci-dessus. Les laits leur ont été distribués à raison de deux repas égaux par jour. Pour des raisons d'ordre sanitaire, tous les animaux ont été soumis à une période de 4 jours de restriction alimentaire correspondant seulement à la satisfaction des besoins d'entretien.

Ensuite, une gamme de plans d'alimentation a été appliquée, permettant, pour les plus élevés, l'obtention de gains de poids vifs de $800 \mathrm{~g}$ par jour du $5^{\mathrm{e}}$ au $15^{\mathrm{e}}$ jour (ingestion de $220 \mathrm{kcal} \mathrm{EM} / \mathrm{j} / \mathrm{kpP}^{0,75}$ ) et de $1400 \mathrm{~g}$ par jour à partir du $15^{\mathrm{e}}$ jour (ingestion de $340 \mathrm{kcal} E M / \mathrm{j} / \mathrm{kgP}^{0,75}$ ). Pour les plans d'alimentation les plus bas, les apports d'EM représentaient $60 \%$ des précédents et les gains de poids vif étaient de $200 \mathrm{~g}$ et $700 \mathrm{~g}$ par jour pour les 2 périodes indiquées précédemment. Les quantités de lait offertes étaient augmentées tous les 2 jours.

\section{Mesures et analyses.}

L'évolution du poids des animaux a été contrôlée par des pesées hebdomadaires effectuées en début d'après-midi.

Les quantités d'aliments effectivement consommées par les animaux ont été contrôlées et des échantillons aliquotes de lait ont été prélevés à chaque repas pendant toute la durée de l'étude, en vue des analyses.

A partir de l'âge de 15 jours, les veaux ont été attachés en cases individuelles et adaptés au port des harnais en vue de la collecte des fèces et de l'urine. Leurs échanges respiratoires ont été mesurés pendant une période de 2 à 3 jours consécutifs, à un âge compris entre 22 et 26 jours, à l'aide de 2 chambres respiratoires du type circuit ouvert, après 3 jours d'adaptation à cet environnement. Les fèces et l'urine ont été récoltées pendant une période de 6 jours.

Le protocole de récolte, de traitement et d'analyse des différents échantillons de lait, de fèces et d'urine (teneur en matière sèche, en énergie et en azote) ainsi que les méthodes de mesure des échanges respiratoires et le calcul des bilans d'énergie ont été décrits antérieurement (Vermorel et al., 1973).

La teneur en lipides des aliments d'allaitement a été contrôlée par extraction après hydrolyse acide, puis pesée des lipides obtenus. Leur composition en acides gras a été déterminée par chromatographie en phase gazeuse sous forme d'esters méthyliques sur colonne capillaire d'acier $(100 \mathrm{~m}$ de long ; $0,5 \mathrm{~mm}$ de diamètre) garnie de carbowax $20 \mathrm{M}$ traité à l'acide téréphtalique. 
Les quantités d'énergie fixée totale $\left(E F^{*}\right)$, sous forme de lipides $\left(L F^{*}\right)$ et sous forme de protéines $\left(\mathrm{PF}^{*}\right)\left(\mathrm{kcal} / \mathrm{j} / \mathrm{kg}^{0,75}\right)$ par les veaux des 4 lots ont été comparées par analyse de covariance multiple (Snedecor et Cochran, 1971) mettant en jeu dans un premier temps les 3 variables suivantes :

a) la quantité d'énergie métabolisable ingérée $\left(\mathrm{EM}^{*}, \mathrm{kcal} / \mathrm{j} / \mathrm{kgP}^{0,75}\right)$;

b) les différences individuelles $\left(\mathrm{Nd}^{*}, \mathrm{mg} / \mathrm{j} / \mathrm{kgP}^{0,75}\right)$ entre la quantité d'azote digestible réellement ingérée et la quantité qui aurait été fournie, pour le même apport $d^{\prime} E M$, si pour chaque animal le régime avait été équilibré au niveau de $8,52 \mathrm{mg} \mathrm{Nd} / \mathrm{kcal}$ EM ;

c) le niveau d'alimentation antérieur $\left(\mathrm{EMa}^{*}, \mathrm{kcal} / \mathrm{j} / \mathrm{kgP}^{0,75}\right)$ observé au cours des 15 jours précédant les mesures.

Dans un second temps, les variables n'exerçant pas d'effet significatif sur l'utilisation de l'énergie ingérée ont été supprimées et l'analyse de covariance a été reprise avec deux variables seulement.

Enfin, nous avons cherché à déterminer le coût respectif de la synthèse des lipides et des protéines à l'aide d'un modèle permettant de prendre en compte les effets du niveau d'alimentation antérieur :

$$
E M^{*}=a P^{*}+b L^{*}+c E M a *+d .
$$

\section{Résultats.}

A) Déroulement de l'expérience. - L'examen de la composition en acides gras des lipides alimentaires (tabl. 1) montre que celle-ci ne correspond pas exactement au protocole. En effet, les acides laurique et myristique représentent seulement $12,7 \%$ des acides gras totaux dans le cas du lait "suif-coprah " (soit $4,6 \%$ de l'énergie totale) contre $22,8 \%$ des acides gras totaux (soit $8,7 \%$ de l'énergie totale) dans le cas du lait "suif-coprah-TC6 ». Cette observation fait supposer que la composition du prémélange réengraissé apportant de I'huile de coprah n'aurait pas été homogène. En revanche, l'acide caproïque représente $34 \%$ des acides gras totaux (soit 10,6 à 10,8\% de l'énergie totale) aussi bien dans le cas du lait " suif-TC6 " que dans le cas du lait « suif-coprahTC6 ».

Les bilans d'énergie obtenus à partir des échanges respiratoires (bilan QR) ou à partir du bilan carbone-azote (bilan $\mathrm{CN}$ ) sont très voisins, les écarts représentant en moyenne 1,2\% seulement de l'énergie fixée soit $0,6 \%$ de l'EM ingérée : nous avons donc utilisé la moyenne des deux valeurs pour exprimer la quantité d'énergie fixée par les veaux.

B) Utilisation digestive et métabolique des régimes. - La digestibilité apparente des aliments est indépendante du niveau d'ingestion. Elle est relativement élevée dans tous les cas, mais présente une variabilité importante (coefficient de variation moyen de $2 \%$ s'élevant jusqu'à 3,6\% dans le cas du lait " suifcoprah $")$. Les coefficients d'utilisation digestive apparents (CUDa) sont du même ordre pour les régimes " suif ", " suif-coprah » et " suif-TC6 ", mais significativement $(P<0,05)$ plus élevés $(+2,8$ ou $+1,9$ points respectivement pour l'énergie et pour l'azote) dans le cas du régime « suif-coprah-TC6 » (tabl. 2). 
TABLEAU 2

Utilisation digestive 'et métabolique des aliments

\begin{tabular}{|c|c|c|c|c|c|c|c|c|c|c|c|}
\hline \multirow[b]{2}{*}{ Nombre d'animaux } & \multicolumn{3}{|c|}{ "Suif $»$} & \multicolumn{3}{|c|}{ "Suif-coprah" } & \multicolumn{3}{|c|}{ «Suif-TC6 » } & \multicolumn{2}{|c|}{ "Suif-coprah-TC6" } \\
\hline & \multicolumn{3}{|c|}{7} & \multicolumn{3}{|c|}{6} & \multicolumn{3}{|c|}{6} & \multicolumn{2}{|l|}{5} \\
\hline CUDa Energie $(\%)$. & $94,4^{a}$ & & 1,8 & $94,2^{a}$ & & 2,6 & $94,9^{a}$ & & 1,6 & $97,3^{b} \pm$ & 0,8 \\
\hline CuDa Azote (\%). & $94,1^{\mathrm{a}}$ & \pm & 1,5 & $93,9^{\mathrm{a}}$ & & 3,4 & $93,6^{\mathrm{a}}$ & & 1,1 & $95,6^{\mathrm{b}} \pm$ & 1,1 \\
\hline EM $\%$ EB $\ldots \ldots \ldots \ldots$ & $91,6^{\mathrm{a}}$ & \pm & 2,0 & $91,6^{a}$ & \pm & 2,8 & $92,1^{\mathrm{a}}$ & \pm & 1,8 & $94,4^{b} \pm$ & 1,0 \\
\hline EM ingérée $\left(\mathrm{kcal} / \mathrm{j} / \mathrm{kg}^{0,75}\right) \ldots$ & 263,3 & \pm & 44,6 & 249,5 & \pm & 36,9 & 225,2 & \pm & 38,5 & $257,2 \pm$ & 52,9 \\
\hline $\mathrm{EMa}\left(\mathrm{kcal} / \mathrm{j} / \mathrm{kg}^{0,75}\right) \ldots \ldots \ldots$ & 252,8 & \pm & 38,3 & 256,2 & \pm & 25,4 & 240,5 & \pm & 38,9 & $254,4 \pm$ & 42,8 \\
\hline $\mathrm{EF}\left(\mathrm{kcal} / \mathrm{j} / \mathrm{kg}^{0,75}\right) \ldots$ & 119,3 & \pm & 25,8 & 122,1 & \pm & 31,4 & 90,3 & \pm & 22,7 & $105,1 \pm$ & $=24,1$ \\
\hline $\mathrm{EF}$ (kcal/kg/gain) (1) ....... & 2243 & \pm & 209 & 2173 & & 241 & 1818 & \pm & 115 & $1734 \pm$ & $=188$ \\
\hline EF ajustée $\left(\mathrm{kcal} / \mathrm{j} / \mathrm{kg}^{0,75}\right)(2)$ & $107,5^{a}$ & \pm & 8,3 & $122,6^{b}$ & \pm & 8,3 & $105,6^{a}$ & \pm & 8,3 & $99,1^{a} \pm$ & 8,3 \\
\hline $\mathrm{LF}\left(\mathrm{kcal} / \mathrm{j} / \mathrm{kg}^{0,75}\right) \ldots \ldots \ldots$ & 67,1 & \pm & 17,0 & 68,5 & \pm & 21,9 & 42,4 & \pm & 11,5 & $45,5 \pm$ & 9,4 \\
\hline LF ajustée $\left(\mathrm{kcal} / \mathrm{j} / \mathrm{kg}^{0,75}\right)(2)$ & $60,4^{a}$ & \pm & 8,7 & $69,0^{\mathrm{a}}$ & \pm & 8,7 & $50,9^{b}$ & \pm & 8,7 & $42,6^{\mathrm{b}} \pm$ & 8,7 \\
\hline $\mathrm{PF}\left(\mathrm{kcal} / \mathrm{j} / \mathrm{kg}^{0,75}, \ldots \ldots \ldots\right.$ & 52,2 & \pm & 9,1 & 53,6 & \pm & 4,8 & 47,9 & \pm & 3,0 & $59,6 \pm$ & 4,9 \\
\hline $\mathrm{PF}$ ajustée $\left(\mathrm{kcal} / \mathrm{j} / \mathrm{kg}^{0.75}\right)(2)$ & $47,6^{a}$ & \pm & 0,4 & $53,6^{b}$ & \pm & 3,2 & $53,8^{b}$ & \pm & 1,6 & $57,5^{c} \pm$ & 2,9 \\
\hline
\end{tabular}

$E F=$ énergie fixée $; L F=$ énergie des lipides fixés ; PF = énergie des protéines fixées ; EM = énergie métabolisable ; $E M a=$ energie métabolisable ingérée au cours des 15 jours anterieurs aux mesures.

a, b, c : les donnees homologues affectées d'exposants différents sont significativement differentes $(P<0,05)$

(1): Données calculées à partir de vitesses de croissance théoriques correspóndant à une fixation de protéines de $170 \mathrm{~g} / \mathrm{kg}$ gain (Van Es et al., 1966).

(2): Données ajustées pour un niveau de $E M=249 \mathrm{kcal} / \mathrm{j} / \mathrm{kg}^{0,75}$, un niveau d'alimentation antérieur $\mathrm{EMa}=251 \mathrm{kcal} / \mathrm{j} / \mathrm{kg}^{0,75}$ et un régime équilibré au niveau de $8,52 \mathrm{mg} \mathrm{Nd} / \mathrm{kcam} \mathrm{EM}$.

Le rapport q entre I'EM et l'énergie totale ingérée est significativement $(P<$ $0,05)$ plus élevé $(+2,8$ points) pour l'aliment " suif-coprah-TC6 » que pour les 3 autres aliments (tabl. 2).

Compte tenu de leur utilisation digestive et métabolique, les 4 régimes présentent des teneurs en azote digestible $(\mathrm{Nd})$ voisines : 8,59 $\pm 0,33 ; 8,36 \pm$ 0,$21 ; 8,59 \pm 0,06$ ou $8,56 \pm 0,12 \mathrm{mg} \mathrm{Nd} / \mathrm{kcal} \mathrm{EM}$, respectivement pour les régimes " suif », "suif-coprah », " suif-TC6 » ou " suif-coprah-TC6 ».

C) Fixation d'énergie dans les tissus. - L'analyse de covariance montre que les différences individuelles de concentration azotée ( $\mathrm{Nd} / \mathrm{EM})$ des rations n'ont pás d'effet sur l'efficacité d'utilisation de I'EM pour la croissance. De plus, celleci n'est pas significativement différente entre les quatre régimes et s'élève en moyenne à $0,70 \pm 0,09$ (fig. 1). Le besoin d'entretien des veaux $(90 \mathrm{kcal} / \mathrm{j} / \mathrm{kgP} 0,75$ en moyenne) est significativement relié au niveau d'alimentation antérieur : il augmente (équation, fig. 1) de $4,6 \mathrm{kcal} / \mathrm{j} / \mathrm{kgP} 0,75$ pour un accroissement $\mathrm{du}$ niveau d'alimentation antérieur de $35 \mathrm{kcal} E M / \mathrm{j} / \mathrm{kgP} 0,75$ qui correspond en pratique à une accélération de la vitesse de croissance de $100 \mathrm{~g} / \mathrm{j}$.

Les quantités moyennes d'énergie fixée ajustées pour un niveau d'ingestion de $249 \mathrm{kcal} E M / \mathrm{j} / \mathrm{kgP}^{0,75}$ et un niveau d'alimentation antérieur de $251 \mathrm{kcal}$ $\mathrm{EM} / \mathrm{j} / \mathrm{kgP}^{0,75}$ (tabl. 2) sont significativement plus élevées avec le régime " suifcoprah » qu'avec le régime "suif-coprah-TC6" $1+24 \%, P<0,001)$ ou qu'avec les deux autres régimes $(+15 \%, P<0,005)$. 


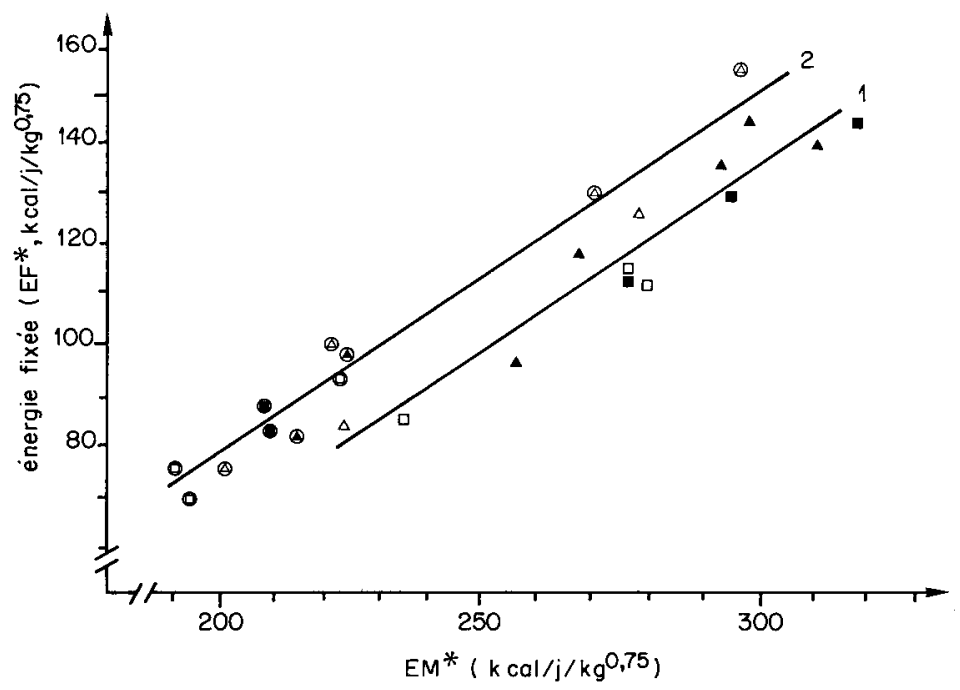

FIG. 1. - Variations de la quantité d'énergie fixée dans l'organisme en fonction de la quantité d'EM ingérée et du niveau d'alimentation antérieur (EMa).

$\mathrm{EF}^{*}=(0,70 \pm 0,09) \mathrm{EM}^{*}-(0,13 \pm 0,06) \mathrm{EMa}^{*}-30,5 \pm 8,3(\mathrm{n}=24 ; \mathrm{R}=0,94)$.

Courbe $\mathrm{n}^{\circ} 1, \mathrm{EMa}^{*}=278 \mathrm{kcal} / \mathrm{j} / \mathrm{kg}^{0,75}$, correspondant à la moyenne observée pour les points non cerclés.

Courbe $\mathrm{n}^{\circ} 2, \mathrm{EMa}^{*}=191 \mathrm{kcal} / \mathrm{j} / \mathrm{kg}^{0,75}$, correspondant à la moyenne observée pour les points entourés d'un cercle.

$\Delta$ : Lait "suif "; $\triangle$ : Lait "suif-coprah" ; $\square$ : Lait "suif-TC6"; $\mathbf{\square}$ : Lait " suif-coprah-TC6".

D) Fixation de lipides dans les tissus. - Comme dans le cas de l'énergie fixée totale, la fixation d'énergie sous forme de lipides $\left(E L^{*}\right) n^{\prime}$ est pas affectée par la concentration azotée $(\mathrm{Nd} / \mathrm{EM})$ des rations. En revanche, elle est reliée à la quantité $d^{\prime} E M^{*}$ ingérée et au niveau $d^{\prime}$ alimentation antérieur $\left(E M^{*} a\right.$ ) selon la régression suivante :

$\mathrm{LF}^{*}=(0,40 \pm 0,09) \mathrm{EM}^{*}-(0,12 \pm 0,08) \mathrm{EMa}^{*}-19,9 \pm 6,8(\mathrm{n}=24 ; r=0,91)$.

Les coefficients de régression ne sont pas significativement différents entre les 4 lots. En revanche, par rapport aux valeurs observées dans le cas du régime " suif », les quantités moyennes ajustées d'énergie fixée sous forme de lipides sont augmentées de $14 \%$ (NS) avec le régime " suif-coprah » ou réduites de $16 \%(\mathrm{P}<0,05)$ ou de $30 \%(\mathrm{P}<0,01)$ respectivement avec les régimes « suifTC6 » ou " suif-coprah-TC6» (tabl. 2).

E) Fixation de protéines dans les tissus. - L'analyse de covariance montre que le niveau d'alimentation antérieur des animaux n'a pas d'effet sur la fixation de protéines (diminution de $1 \pm 3 \mathrm{kcal} / \mathrm{kgP}^{0,75}$ pour une réduction de $100 \mathrm{kcal}$ $\left.\mathrm{d}^{\prime} \mathrm{EMa}^{*}, \mathrm{P}>0,50\right)$.

Les différences individuelles de concentration azotée (Nd/EM) des rations exercent un effet semblable pour les quatre régimes, soit un accroissement de 
2,25 kcal de protéines fixées (63 $\mathrm{mg}$ d'azote fixé) pour une augmentation de $100 \mathrm{mg}$ de la quantité d'azote digestible ingéré, à quantité de EM ingérée identique.

Les quantités d'énergie fixées sous forme de protéines $\left(P F^{*}\right)$ sont reliées aux quantités $\mathrm{d}^{\prime} \mathrm{EM}$ ingérées $\left(E \mathrm{E}^{*}\right)$ selon des régressions linéaires de pentes respectives $0,25 \pm 0,01 ; 0,26 \pm 0,05 ; 0,30 \pm 0,04$ et $0,29 \pm 0,05$ pour les régimes "suif ", " suif-coprah ", " suif-TC6" ou " suif-coprah-TC6" (fig. 2). Ces pentes sont significativement différentes pour les régimes "suif » et "suif-TC6 " $(P<0,025)$ tandis que la différence observée entre les régimes " suif » et " suifcoprah-TC6 " dépasse le seuil de probabilité de $90 \%(P<0,10)$.

Les quantités d'énergie fixées sous forme de protéines, ajustées pour les mêmes quantités d'EM et d'azote digestible, sont supérieures de 13,2 \% $(\mathrm{P}<0,05)$ dans le cas des régimes "suif-coprah " et " suif-Tri C6 " et de $20,8 \%(P<0,001)$ dans le cas du régime « suif-coprah-Tri C6 " par rapport au

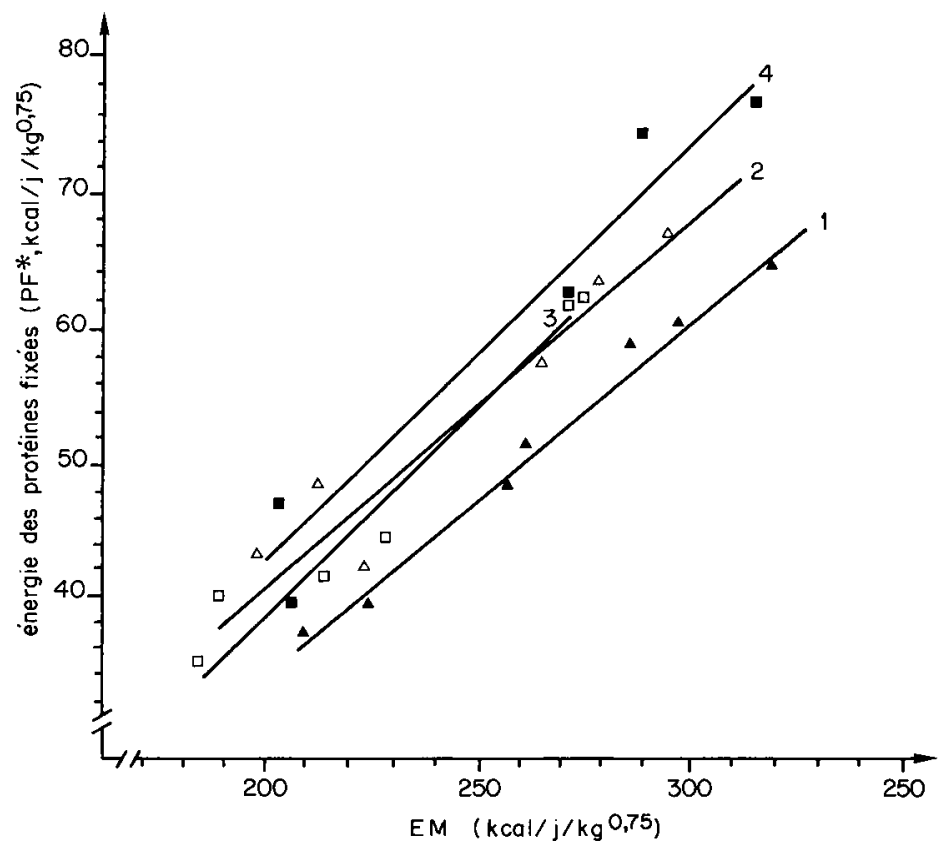

FIG. 2. - Variations de la quantité d'énergie fixée sous forme de protéines en fonction de la quantité $d^{\prime} E M$ ingérée selon la nature du régime.

Lait " suif "

: Courbe $n^{\circ} 1, \mathrm{PF}^{*}=(0,25 \pm 0,01) \mathrm{EM}^{*}-14,7 \pm 0,6$

Lait « suif-coprah "

( $\mathrm{n}^{-}=7 ; r=0,99$ )

Lait « suif-TC6 »

: $\triangle$, Courbe $n^{\circ} 2, \mathrm{PF}^{*}=(0,26 \pm 0,05) \mathrm{EM}^{*}-6,1 \pm 3,2$

(n $=6 ; r=0,95)$

Lait " suif-coprah-TC6 »

: $\square$, Courbe $n^{\circ} 3, \mathrm{PF}^{*}=(0,30 \pm 0,04) \mathrm{EM}^{*}-18,8 \pm 1,8$

$$
\text { (n }=6 ; r=0,92 \text { ) }
$$

口. Courbe $n^{\circ} 4, \mathrm{PF}^{*}=(0,29 \pm 0,06) \mathrm{EM}^{*}-14,8 \pm 3,0$ 
régime "suif " (tabl. 2). Les effets favorables de l'huile de coprah et de la tricaproïne sont partiellement additifs puisque les quantités de protéines fixées sont supérieures de 8,1 et $7,6 \%(P<0,05)$ avec le régime 4 par rapport aux régimes 2 et 3 respectivement.

\section{Discussion.}

A) Utilisation digestive des aliments. - Dans cette étude, le remplacement d'une partie du suif de l'aliment témoin par des acides gras à chaîne moyenne (tricaproïne, C12 et C14 de I'huile de coprah) n'a pas entraîné d'amélioration des CUDa de l'énergie et de l'azote contrairement aux résultats obtenus précédemment avec du lait entier (Mathieu et Barre, 1964 ; Raven et Robinson, 1958) ou des laits enrichis en huile de coprah (Toullec et Mathieu, 1969; Veen, 1970a ; Roy et al., 1973). La faible teneur en acides laurique et myristique $(2,8 \%$ de la MS) du lait « suif-coprah » peut expliquer cette contradiction, mais cette explication n'est pas valable dans le cas du lait « suif-Tri C6" contenant $7,2 \%$ de la matière sèche sous forme d'acide caproïque.

En revanche, dans le cas du lait " suif-coprah-TC6 ", la très forte proportion des acides gras à chaîne moyenne $(12,2 \%$ de la MS ou $56,8 \%$ des acides gras totaux) a permis une augmentation significative de l'utilisation digestive de l'énergie du lait. Pour cet aliment, la digestibilité des matières azotées est également améliorée comme cela avait été observé précédemment dans le cas de laits enrichis en huile de coprah (Roy et al., 1973). Il a été démontré que, chez le Veau préruminant, les acides gras à chaîne moyenne étaient activement libérés dans la caillette sous l'action des lipases prégastriques (Siewert et Otterby, 1968; Edwards-Webb et Thompson, 1978), $66 \%$ de I'acide caproïque étant absorbés directement au niveau de la caillette (Edwards-Webb et Thompson, 1978). Le caillot formé par la coagulation des caséines du lait est donc appauvri en globules gras et l'attaque des protéines dans la caillette peut être facilitée (Ternouth et al., 1975), ce qui expliquerait la meilleure utilisation digestive des matières azotées.

B) Fixation d'énergie dans les tissus. - La diminution des dépenses énergétiques chez des animaux soumis à des plans d'alimentation restrictifs est un phénomène qui retient de plus en plus l'attention des chercheurs (Thorbek et Henckel, 1976 chez le bovin, par exemple). La prise en compte du produit $\left(\mathrm{EMa}^{*} \times\right.$ $\mathrm{EM}^{*}$ ) lors de l'analyse des résultats par régression multiple ne réduit pas significativement l'écart type résiduel $(P>0,50)$, ce qui exclut un effet du niveau d'alimentation antérieur $\left(\mathrm{EMa}^{*}\right.$ ) sur le rendement de I'utilisation de $\mathrm{EM}^{*}$ pour la croissance. Les résultats rapportés ci-dessus montrent donc que les effets d'une réduction du plan d'alimentation portent en totalité sur le besoin d'entretien ; de plus, sa diminution est compensée pour $92 \%$ par un accroissement de la fixation de lipides et pour $8 \%$ seulement par un accroissement (non significatif) de la fixation de protéines. 
L'accroissement important de la fixation de lipides dans le cas de l'aliment " suif-coprah" ( $14 \%$ en moyenne) est en accord avec les observations de Roy et al. (1973). Dans les présents résultats, il pourrait être lié en partie à un effet curvilinéaire du niveau d'alimentation antérieur sur l'utilisation de l'énergie mais l'analyse statistique ne permet pas de retenir cette hypothèse. Bien que cet accroissement ne soit pas significatif, il fausse la détermination du coût respectif de la protéinogenèse et de la lipogenèse.

Le calcul repris en èliminant les données relatives à l'utilisation de l'aliment « suif-coprah » et en prenant en compte l'effet du niveau d'alimentation antérieur conduit à des résultats plus cohérents $(P<0,001)$ :

$\mathrm{EM}^{*}=(2,29 \pm 0,21) \mathrm{PF}^{*}+(0,99 \pm 0,15) \mathrm{LF}^{*}+(0,13 \pm 0,05) \mathrm{EMa}^{*}+43,1 \pm 9,1$

( $n=18: r=0,98$ )

Ce calcul montre, comme celui concernant la fixation globale d'énergie dans les tissus, un accroissement des besoins d'entretien des animaux de $13 \mathrm{kcal}$ pour une augmentation des quantités de EM ingérées de $100 \mathrm{kcal}$. Le coût de la fixation des lipides ainsi estimé $(0,99 \pm 0,15 \mathrm{kcal} E M / \mathrm{kcal} L F)$ pourrait indiquer une fixation exclusive d'acides gras longs alimentaires. L'efficacité d'utilisation de I'EM pour la fixation de protéines $(43 \%)$ est identique aux valeurs observées antérieurement chez le Veau (Kirchgessner et al., 1976) tandis que l'efficacité de fixation des lipides est meilleure. Le besoin de 1,17 kcal EM/kcal LF observé par ces auteurs pourrait correspondre à l'emploi d'animaux plus âgés pour lesquels la part des acides gras synthétisés deviendrait plus importante.

A partir des coûts de fixation des protéines et des lipides ainsi déterminés, on peut estimer avec une incertitude de 6 points l'efficacité d'utilisation de EM pour la croissance à 67,4 - 67,5 - 64,4 et 60,5\% respectivement pour les régimes " suif ", "suif-coprah ", "suif-TC6 " et "suif-coprah-TC6 ». Cette approche conduit à une estimation des besoins d'entretien sensiblement plus faible que la précédente $\left(85\right.$ contre $\left.90 \mathrm{kcal} / \mathrm{j} / \mathrm{kgP}^{0,75}\right)$.

Enfin, la vitesse de croissance des veaux, mesurée dans cette expérience sur des périodes trop courtes pour être valable, peut être estimée indirectement à partir de la quantité de protéines fixées dans les tissus en admettant une fixation de $170 \mathrm{~g}$ de protéines par $\mathrm{kg}$ de gain (Van Es et al., 1966). Dans ces conditions, les quantités d'énergie fixées par $\mathrm{kg}$ de gain (tabl. 2) sont du même ordre pour les animaux des lots " suif » et " suif-coprah » $2243 \pm 209$ et $2173 \pm 241 \mathrm{kcal}$ respectivement). Elles sont réduites de $20 \%$ dans le cas des animaux des lots " suif-TC6 " et " suif-coprah-TC6 » $1818 \pm 115$ et $1734 \pm 181$ kcal respectivement).

C) Fixation de protéines. - Différents auteurs avaient mis en évidence que I'efficacité d'utilisation des aliments pour la croissance pouvait être améliorée par I'utilisation d'huile de coprah (Raven et Robinson, 1964 ; Veen, 1970a ; Roy et al., 1973) ou de triglycérides d'AGCM synthétiques de 6 à 10 carbones (Namiotkevicz, 1973). Nos résultats, en accord avec ceux de Veen (1970a), montrent que les effets favorables des AGCM sur la croissance correspondent à une amélioration de l'utilisation des matières azotées ingérées pour la fixation de protéines dans les tissus, contrairement aux observations de Roy et al. (1973). Les effets 
favorables des $A G C M$ sur la protéinogenèse (PF) se traduisent par une augmentation de la proportion d'énergie fixée sous forme de protéines qui passe de $43,8 \%$ pour les régimes "suif 》 et "suif-coprah " à $51,8 \%$ pour le régime " suif-TC6 " et à $57 \%$ pour le régime " suif-coprah-TC6".

\section{Conclusions.}

Les résultats obtenus au cours de cette expérience ont permis de montrer que l'incorporation d'acides gras à chaîne moyenne aux aliments du jeune Veau préruminant permet de mieux extérioriser ses potentialités de protéinogenèse et de croissance. Les effets portent à la fois sur l'utilisation digestive de l'énergie et des matières azotées ingérées et sur l'efficacité d'utilisation de ces dernières pour la fixation de protéines dans les tissus.

Ces résultats montrent que par rapport à I'utilisation des matières grasses conventionnelles, l'emploi des acides gras à chaîne moyenne, peut permettre une augmentation de plus de $22 \%$ de la fixation de protéines aux dépens de la fixation de lipides et un accroissement correspondant au gain de poids vif des veaux préruminants. Les acides gras à chaîne moyenne sont donc particulièrement bien adaptés à l'alimentation des veaux au cours des premiers jours et des premières semaines de leur vie et à la couverture de leurs besoins énergétiques pendant cette période.

Reçu en mai 1982.

Accepté en janvier 1983.

\section{Références}

CHENAT M. C., AUROUSSEAU B., VERMOREL M., 1976. Influence de I'ingestion d'acide caproïque sur l'utilisation de l'énergie et de l'azote du régime par le rat en croissance en fonction de l'âge des animaux et de la teneur en azote des régimes. Ann. Biol. anim. Bioch. Biophys., 16, 603-622.

EDWARDS-WEBB J. D., THOMPSON S. Y., 1978. Studies on lipid digestion in the preruminant calf. 3. The action of salivary lipase on milk fat in the abomasum. Brit. J. Nutr., 40, 125131.

ES A. J. H. Van, WEERDEN E. J. Van, FRENS A. M., 1966. Wärmeproduktion, Wasserverdünstungs und Energiestoffwechsel bei Mastkälbern, gefüttert mit einem flüssigen Milchersatzfutter. Zeitsch. Tierphysiol. Tierernähr., Futterm., 21, 25-33.

KIRCHGESSNER M., MULLER H. L., NEESSE K. R., 1976. Energy retention and utilization by the veal calf. In M. VERMOREL, Energy metabolism of farm animals, EAAP no 19, 201-204.

LODGE G. A., LISTER E. E., 1973. Effects of increasing the energy value of a whole milk diet for calves. I. Nutrient digestibility and nitrogen retention. Can. J. anim. Sci., 53, 307-316.

MATHIEU C. M., BARRE P. E., 1964. Digestion et utilisation des aliments par le veau préruminant à l'engrais. I. utilisation des laits entiers ou partiellement écrémés. Ann. Biol. anim. Bioch. Biophys., 4, 403-422. 
MATHIEU C. M., DE TUGNY M., 1965. Digestion et utilisation des aliments par le veau préruminant à l'engrais. II. Remplacement des matières grasses du lait par du glucose. Ann. Biol. anim. Bioch. Biophys., 5, 21-39.

MATHIEU C. M., THIVEND P., 1968. Digestion et utilisation des aliments par le veau préruminant à l'engrais. II. Remplacement des matières grasses du lait par différents amidons. Ann. Biol. anim. Bioch. Biophys., 5, 249-271.

NAMIOTKEVICZ J., 1973. L'utilisation des acides gras synthétiques pour l'engraissement des veaux. Rocz. Nauk. Roln., 394, 7-16.

NIESAR K. M., 1964. Quälitätsmerkmale und Quälitätsbewertung der Fettkcomponente in Milschfutter. Bayer. Landw. Jb., 41, 230-242.

RAVEN A. M., ROBINSON K. L., 1958. Studies of the nutrition of the young calf. 1. Comparison of starch, lactose and hydrogenated palm oil, with butterfat in milk replacers. Brit. J. Nutr., 12, 469-482.

RAVEN A. M., ROBINSON K. L., 1964. Factors affecting the nutrition value of fat for calves. 1. The mode of incorporation into milk diets. J. Sci. Food Agric., 15, 214-219.

ROY J. M. B., STOBO I. J. F., GASTON H. J., SHOTTON S. M., GANDERTON P., 1973. The nutrition of the veal calf. 6 . The effect of ultra high $(68 \%)$ fat milk powders added to liquid skim-milk and a comparison with spray dried skim milk powder containing $20 \%$ margarine fat. Anim. Prod., 17, 109-127.

SIEWERT K. L., OTTERBY D. E., 1968. Lipolysis of milk fat by esterase pregastric in vivo. J. Dairy Sci, 51, 1305-1307.

SNEDECOR G. W., SNEDECOR W. G., 1971. Méthodes statistiques. Ass. Coord. Tech. Agric. ed., Paris, 488-496.

STOBO I. J. F., ROY J. H. B., 1977. The use of microbal protein in milk substitute diets for calves. Proc. Brit. Soc. anim. Prod., 24, 143A.

TERNOUTH J. H., ROY J. H. B., THOMPSON S. Y., TOOTHILL J., GILLIES C. M., EDWARDS WEBB J. D., 1975. Concurrent studies of the flow of digesta in the duodenum and exocrine pancreatic secretion of calves. 3. Further studies on the addition of fat to skim-milk and the use of non milk proteins in milk substitute diets. Br. J. Nutr., 31, 181-196.

THORBEK G., HENCKEL S., 1976. Studies on energy requirement for maintenance in farm animals. In M. VERMOREL, Energy metabolism of farm animals, EAAP $\mathrm{n}^{\circ} 19,117-120$.

TOULLEC R., MATHIEU C. M., 1969. Utilisation digestive des matières grasses et de leurs principaux acides gras par le veau préruminant à l'engrais. Influence sur la composition corporelle. Ann. Biol. anim. Bioch. Biophys., 9, 139-160.

VEEN W. A. G., 1970a. Animal and vegetable fats in milk replacers for veal calves. 1. Digestibility coefficients of fat and nitrogen retention. Zeitsch. Tierphysiol. Tierernähr. Futterm., 26, 264-279.

VEEN W. A. G., 1970b. Animal and vegetable fats in milk replacers for veal calves. III. A comparison of two methods of extracting faecal fat on the digestibility coefficients of fatty acids. Zeitsch. Tierphysiol. Tierernähr. Futterm., 27, 193-202.

VERMOREL M., BOUVIER J. C., BONNET Y., FAUCONNEAU G., 1973. Construction et fonctionnement de 2 chambres respiratoires du type " circuit ouvert " pour jeunes bovins. Ann. Biol. anim. Bioch. Biophys., 13, 659-681. 